\title{
Bioindicadores de Poluição Ambiental: Um Estudo Bibliométrico
}

\author{
Bioindicators of environmental pollution: A Bibliometric Study
}

Bioindicadores de contaminación ambiental: Un Estudio Bibliométrico

\section{Sofia Negri Braz}

Graduada em Ciências Biológicas e Mestranda em Sustentabilidade pela Pontifícia Universidade Católica de Campinas, Campinas, SP soh_braz@hotmail.com

\section{Regina Márcia Longo}

Professora e pesquisadora em dedicação integral da Pontifícia Universidade Católica de Campinas e membro do corpo permanente de docentes dos cursos de mestrado em Sistemas de Infraestrutura Urbana (PUC-Campinas) e Sustentabilidade (PUC-Campinas). regina.longo@puc-campinas.edu.br 


\section{ANAP

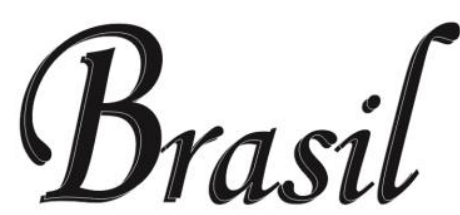 \\ ISSN 1984-3240 v. 12, n. 27}

\section{RESUMO}

Algumas atividades antrópicas têm contribuído de maneira efetiva para o aumento significativo de contaminantes nos ecossistemas, permitindo que estes se tornem disponíveis na água, solo e atmosfera. Além das avaliações convencionais físico-químicas de poluição ambiental, o uso de bioindicadores também é uma metodologia adequada para a detecção desses poluentes. Este recurso consiste em um grupo de espécies ou comunidades biológicas cuja quantidade, distribuição e presença indicam a magnitude de impactos ambientais em um ecossistema. Diante disso, o presente estudo teve como objetivo realizar um levantamento bibliométrico sobre bioindicadores de poluição ambiental a partir de documentos eletrônicos publicados com o intuito de identificar como o tema tem sido abordado no âmbito acadêmico no período de 2008 à 2018. Foram analisados um total de 70 amostras do Portal CAPES e BDTD, as quais foram, posteriormente, divididas por categorias de publicações, como: ano, região do Brasil, documento, metodologia, bioindicador e aplicação do bioindicador. Com os resultados obtidos observou-se que em o maior número de publicações por ano aconteceu em 2016, o local de maior publicação foi a região Sudeste, o tipo de metodologia mais utilizada foi a científica e o tipo de documento mais encontrado foi em forma de Artigo. Com relação aos bioindicadores, dos 70 estudos coletados, 48,6\% utilizaram animais como indicadores biológicos e o mesmo percentual se aplicou ao elemento que mais preocupa os estudiosos: a água. Concluiu-se que a ferramenta utilizada no presente trabalho possibilita o entendimento mais amplo sobre o tema abordado de forma a contribuir em produções científicas subsequentes.

PALAVRAS-CHAVE: Levantamento Bibliomátrico. Poluição Ambiental. Bioindicadores.

\section{ABSTRACT}

Some anthropic activities have effectively contributed to the significant increase of contaminants in ecosystems, allowing them to become available in water, soil and atmosphere. In addition to the conventional physical-chemical evaluations of environmental pollution, the use of bioindicators is also an adequate methodology for the detection of these pollutants. This resource consists of a group of biological species or communities whose quantity, distribution and presence indicate the magnitude of environmental impacts in an ecosystem. Therefore, the present study aimed to carry out a bibliometric survey on bioindicators of environmental pollution from electronic documents published with the intention of identifying how the subject has been approached in the academic field from 2008 to 2018. A total of 70 samples of CAPES Portal and BDTD, which were later divided into categories of publications, such as: year, region of Brazil, document, methodology, bioindicator and bioindicator application. With the results obtained it was observed that in the largest number of publications per year occurred in 2016, the place of greatest publication was the Southeast region, the type of methodology most used was the scientific one and the type of document most found was in the form of Article. Regarding the bioindicators, of the 70 studies collected, 48.6\% used animals as biological indicators and the same percentage was applied to the element that most worries the students: water. It was concluded that the tool used in the present work makes possible a broader understanding of the topic in order to contribute to subsequent scientific productions.

KEYWORDS: Bibliometric Survey. Environment pollution. Bioindicators.

\section{RESUMEN}

Algunas actividades antrópicas han contribuido de manera efectiva al aumento significativo de contaminantes en los ecosistemas, permitiendo que éstos se hagan disponibles en el agua, el suelo y la atmósfera. Además de las evaluaciones convencionales físico-químicas de contaminación ambiental, el uso de bioindicadores también es una metodología adecuada para la detección de esos contaminantes. Este recurso consiste en un grupo de especies o comunidades biológicas cuya cantidad, distribución y presencia indican la magnitud de impactos ambientales en un ecosistema. El presente estudio tuvo como objetivo realizar un levantamiento bibliométrico sobre bioindicadores de contaminación ambiental a partir de documentos electrónicos publicados con el propósito de identificar cómo el tema ha sido abordado en el ámbito académico en el período de 2008 a 2018. Se analizaron un total De acuerdo con lo establecido en la Ley Orgánica 15/1999 de la Comisión de las Comunidades Europeas. Con los resultados obtenidos se observó que en el mayor número de publicaciones al año se produjo en 2016, el lugar de mayor publicación fue la región Sudeste, el tipo de metodología más utilizada fue la científica y el tipo de documento más encontrado fue en forma de " artículo. Con respecto a los bioindicadores, de los 70 estudios recolectados, el 48,6\% utilizaron animales como indicadores biológicos y el mismo porcentaje se aplicó al elemento que más preocupa a los estudiosos: el agua. Se concluyó que la herramienta utilizada en el presente trabajo posibilita el entendimiento más amplio sobre el tema abordado para contribuir en producciones científicas subsiguientes.

PALABRAS CLAVE: Levantamiento Bibliomátrico. Contaminación Ambiental. Bioindicadores. 


\section{ANAP

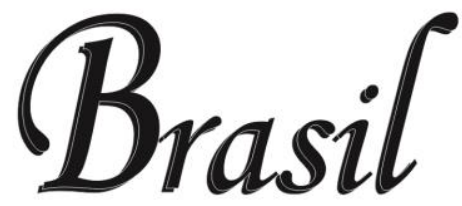 \\ ISSN 1984-3240 V. 12, n. 27}

\section{Introdução}

Atualmente, nosso planeta enfrenta uma grave crise ambiental e isso decorre, principalmente, da ação do homem (TORGAL; JALALI, 2007). Segundo o Ministério do Meio Ambiente, a capacidade de suporte de um ambiente é reconhecida como uma habilidade do mesmo em acomodar, assimilar e incorporar um conjunto de atividades antrópicas sem que suas funções naturais sejam alteradas (BRASIL, 2019). Este conceito tem uma enorme significância em termos das relações entre o processo econômico e o ecossistema, garantindo uma qualidade de vida e ambiental (CAVALCANTI; BEGOSSI, 1997).

A verificação da qualidade ambiental das cidades é cada vez mais importante e evidente com a justificativa de que é no espaço urbano onde os problemas ambientais geralmente atingem maior amplitude. Dentre esses, destaca-se o consumo exagerado de bens materiais, a produção constante de lixo, a degradação do solo e subsolo, em consequência do uso intensivo do território pelas atividades urbanas, a alta concentração de poluentes no ar e na água, entre outros (LOMBARDO, 1985). Por isso, ações motivadas por "valores ambientais", têm sido vistas como uma nova estratégia para garantir um desenvolvimento socioeconômico cada vez mais sustentável e ambientalmente seguro e qualificado (PORTILHO, 2006).

Alguns dos elementos tóxicos sempre foram encontrados e ocorreram de forma natural no meio ambiente (CARDODO et. al., 2017). Contudo, algumas atividades sociais têm contribuído de maneira efetiva para o aumento significativo desses contaminantes em distintos ecossistemas, permitindo que estes se tornem disponíveis em recursos hídricos, solos e atmosfera. Tal problemática socioambiental é um fato que deve ser investigado, visto que, ao depender das concentrações de poluentes em contato com o organismo, proporciona diversos riscos à saúde devido à exposição (SATARUG et al., 2010).

Sendo assim, além das avaliações convencionais físico-químicas de poluição ambiental, o uso de bioindicadores também é uma metodologia adequada para a detecção destes poluentes. 0 termo "bioindicador" é definido como um grupo de espécies ou comunidades biológicas cuja quantidade, distribuição e presença indicam a magnitude de impactos ambientais em um ecossistema (CALISTO; GONÇALVES; MORENO, 2018) modificando suas funções vitais, ou sua composição química (KAPUSTA, 2008). Sua utilização permite a avaliação integrada dos efeitos ecológicos causados por múltiplas fontes de poluição (CALISTO; GONÇALVES; MORENO, 2018). A utilização de organismos como indicadores ambientais vem de longa data, no entanto, a bioindicação começou a ser utilizado mais frequentemente a partir dos anos 1960 (LIMA, 2001).

São inúmeras as vantagens de se usar os bioindicadores como indicação de qualidade ambiental, no geral. Atestar o impacto da poluição sobre um ecossistema, fornecer informações sobre as causas e fatores observados, demonstrar a distribuição espacial e temporal do impacto e fornecer dados sobre um potencial risco para a flora, fauna e população humana foram algumas das vantagens citadas por KAPUSTA (2008), em seu livro publicado em 2008 sobre bioindicação (KAPUSTA, 2008). Além disso, seu baixo custo ainda possibilita que esse recurso seja utilizado para avaliação cumulativa de eventos ocorridos num 


\section{ANAP

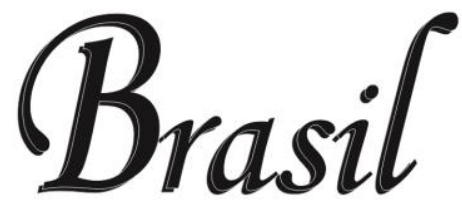 \\ ISSN 1984-3240 v. 12, n. 27}

determinado período de tempo, resgatando um histórico ambiental não passível de detecção ou medição por outros métodos (FIALHO, 2002). CARNEIRO, 2004 ainda destaca que outra vantagem é que a metodologia não exige, em geral, de aparelhagem sofisticada de medição, viabiliza a avaliação de elementos químicos presentes em baixas concentrações no ambiente em estudo e é visto como eficiente no monitoramento de áreas amplas. Porém, vale ressaltar que, os indicadores biológicos não podem realizar sozinhos a caracterização da poluição, pois os mesmos servem para indicar a presença do poluente mas não faz a quantificação da concentração do mesmo (CARNEIRO, 2004).

Alguns autores, ainda, ressaltam que há uma dificuldade em diferenciar, nos organismos, os efeitos dos poluentes e processos de reação à demais condições ambientais (solo, clima e alterações antrópicas). Assim, o biomonitoramento torna-se mais adequado quando fornece dados que podem ser comparados com informações obtidas pelos métodos convencionais de monitoramento ambiental (KLUMPP et. al. 2001; NIMIS et. al. 2000).

A seleção do bioindicador é baseada nas metas e objetivos do estudo, para uma região ou ecossistema em particular, e na atividade que está sendo analisada (CAIRNS; McCORMICK; NIEDERLEHNER, 1993). Destaca-se que os tipos de bioindicadores variam desde microrganismos, até macrorganismos.

Diante do exposto, é notável o vasto número de estudos relacionados à bioindicação com diversos tipos de bioindicadores e, consequentemente, diversas aplicações dos mesmos. Por isso, faz-se necessário um levantamento de pesquisas publicadas sobre o assunto para gerar uma relação entre a eficiência destes organismos com ambientes poluídos identificando déficits de pesquisas em determinadas categorias.

\section{Objetivos}

Nesse contexto, o presente trabalho teve como objetivo realizar um levantamento bibliométrico sobre bioindicadores de poluição ambiental a partir de documentos eletrônicos publicados com o intuito de identificar como o tema tem sido abordado no âmbito acadêmico.

\section{Metodologia}

Visando alcançar um número representativo da produção científica acerca da temática de Bioindicadores de poluição ambiental, foi realizada busca eletrônica em dois diferentes locais: Portal Periódicos da CAPES e BDTD (Biblioteca Digital de Teses e Dissertações), onde, na primeira foram buscados artigos científicos publicados em diferentes revistas e, o segundo, apenas publicações de teses e dissertações. $O$ período abrangido pela busca foi compreendido entre os anos de 2008 e 2018 e a palavra chave utilizada foi "Bioindicadores de poluição ambiental", onde só foram procurados documentos em português.

Após a organização do material obtido, as referências de cada artigo (autor, título, fonte, ano de publicação) foram organizadas numa tabela e os textos repetidos foram descartados. Dessa forma, o presente estudo contou com a análise de um total de 70 textos à serem analisados. Quando os documentos geravam dúvida quanto ao enquadramento no tema escolhido, foi 
realizada a leitura do resumo/abstract e metodologia dos mesmos.

O material amostrado foi organizado segundo ano de publicação (2008 à 2018), regiões do Brasil (Norte, Nordeste, Centro-Oeste, Sudeste ou Sul), tipo de documento (artigo, tese ou dissertação), tipo de metodologia (científica, revisão de literatura ou estudo de caso), tipo de bioindicador (animal, vegetal, microrganismo, líquen ou animal e vegetal) e aplicação do bioindicador (água, solo, ar ou água solo e ar).

\section{Resultados e Discussão}

Observou-se, no Gráfico 1, que o ano de 2016 foi o ano que apresentou o maior número de publicações (13), e nos anos subsequentes (2017 e 2018) houve um decréscimo na quantidade de documentos sobre a temática, mas acabaram se mantendo estáveis com 6 publicações, assim como os anos de 2013 e 2014, tendo 5 em cada. Destaca-se que o número de artigos publicados em 2015 é, notavelmente, inferior em comparação ao ano com maior publicação, tendência que poderá ser mais profundamente analisada através de estudos posteriores.

Baseado no estudo bibliométrico realizado por BHONA, LOURENÇO e BRUM (2011), a hipótese a ser investigada é a de que em 2016, não somente o interesse acadêmico aumentou, mas, também, que podem ter ocorrido pesquisas com assuntos mais amplos e mais viáveis para estudos. A partir dessa hipótese, um estudo encontrado, feito por FACURY (2019), afirmou que o grande aumento de publicações em 2016 ocorreu, principalmente, devido ao desastre ambiental causado pelo rompimento da barragem do município de Bento Rodrigues, em Mariana - MG em 2015, que destruiu completamente o Rio Doce e toda vida que existia ali, despertando, então, maior interesse e preocupação com o meio ambiente entre os pesquisadores de 2016 até o presente ano.

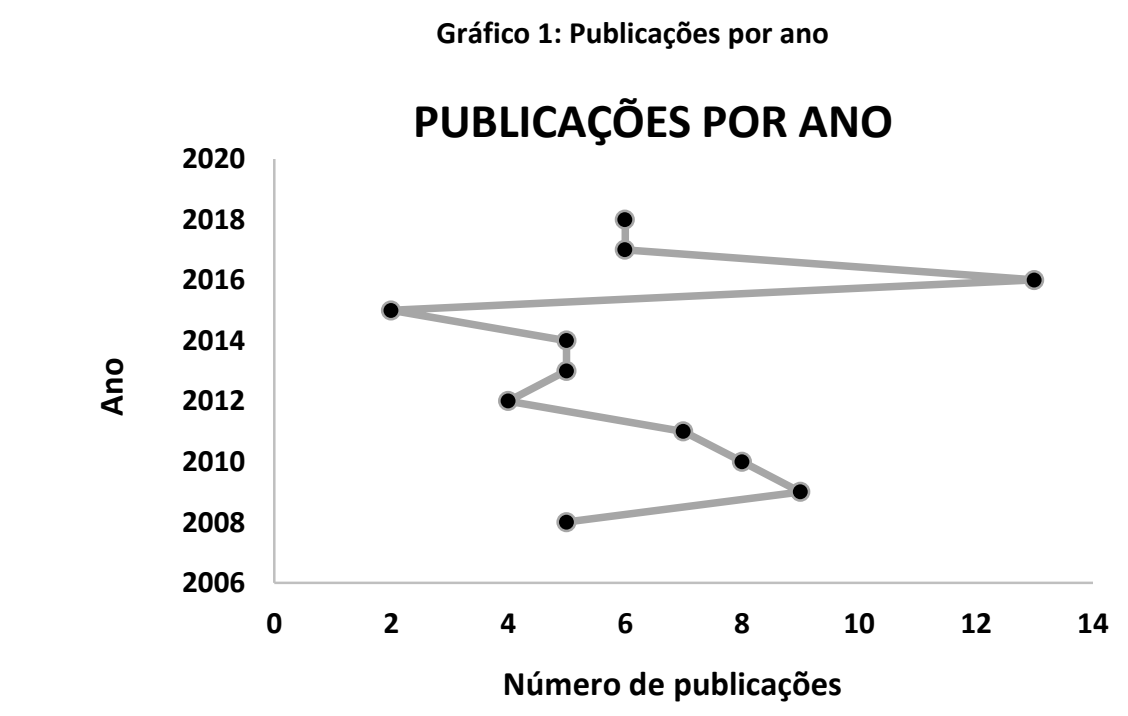

Fonte: Obtidos pelo levantamento no Portal Periódico CAPES e BDTD, adaptado pelo autor, 2019.

Um dos tópicos levantados no estudo foi o de publicações por região do país. No Brasil, se 


\section{ANAP

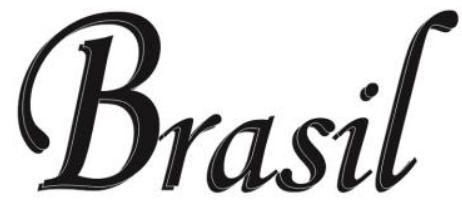 \\ ISSN 1984-3240 v. 12 , n. 27}

verifica uma enorme heterogeneidade espacial das atividades de pesquisa científica, onde o padrão regional da distribuição das publicações e dos pesquisadores é altamente concentrado na região Sudeste, com destaque às capitais dos estados (SIDONI; HADDAD; MENA-CHALCO, 2016) por isso, na presente pesquisa, foi levantado que, de 70 artigos, a região Sudeste foi a que se destacou com, aproximadamente, $48,5 \%$ desse total.

No outro extremo, as regiões Centro-Oeste e Norte foram as que apresentaram menor percentual de publicações, com, aproximadamente, $8,57 \%$ das amostras, enquanto que as regiões Nordeste e Sul se mostraram iguais, com 12 publicações, de acordo com a Tabela 1.

Segundo SIDONI, HADDAD e MENA-CHALCO (2016), a proximidade geográfica aparenta ser um dos principais fatores que contribuem para a ocorrência de interações colaborativas, na medida em que as participações das parcerias intrarregionais são bastante elevadas para todas as regiões. Isso explica, também, o número alto de publicações na região Sudeste, visto que essas "parcerias intrarregionais" entre Rio de Janeiro, São Paulo e Minas Gerais são fortes, enquanto que nas outras, não.

Tabela 1: Publicações por regiões do Brasil

\begin{tabular}{ccc}
\hline REGIÃO DO BRASIL & No DE PUBLICAÇÕES & PERCENTUAL \% (aprox.) \\
\hline Norte & 6 & 8,57 \\
Nordeste & 12 & 17 \\
Centro Oeste & 6 & 8,57 \\
Sudeste & 34 & 48,5 \\
Sul & 12 & 17 \\
\hline
\end{tabular}

Fonte: Obtidos pelo levantamento no Portal Periódico CAPES e BDTD, adaptado pelo autor, 2019.

Com relação aos tipos de metodologia das amostras, observou-se que as publicações com metodologias científicas, ou seja, que apresentam métodos práticos e técnicas para um determinado objetivo fizeram parte da grande maioria de amostras encontradas, totalizando uma porcentagem aproximada de $75,7 \%$, enquanto que estudos de caso e textos de revisão bibliográfica foram, evidentemente, menores, como representados na Tabela 2.

Tabela 2: Tipos de metodologia utilizada

\begin{tabular}{ccc}
\hline METODOLOGIA & No DE PUBLICAÇÕES & PERCENTUAL \% (aprox.) \\
\hline Científica & 53 & 75,7 \\
Revisão Bibliográfica & 11 & 15,7 \\
Estudo de Caso & 6 & 8,6 \\
\hline
\end{tabular}

Fonte: Obtidos pelo levantamento no Portal Periódico CAPES e BDTD, adaptado pelo autor, 2019.

Define-se uma Revisão Bibliográfica como um método científico para busca e análise de artigos de uma determinada área da ciência. A sua importância se da, pois ela delimita uma linha limítrofe da pesquisa que se deseja desenvolver, considerando uma perspectiva científica. Além disso, ela contribui para o desenvolvimento de uma base sólida de conhecimento, facilitando o desenvolvimento da teoria em áreas onde já existem pesquisas, e também, 


\section{ANAP

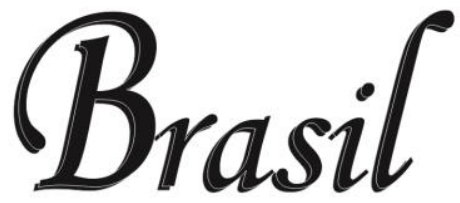 \\ ISSN 1984-3240 v. 12, n. 27}

identificando áreas onde há oportunidades para novas pesquisas (WEBSTER; WATSON, 2002). Com o menor número de publicações, a posição tomada pela Conferência de Cambridge define Estudo de Caso como um termo amplo que inclui "uma família de métodos de pesquisa cuja decisão comum é um enfoque numa instância", podendo ser um evento, uma pessoa, um grupo, entre outros, buscando uma descoberta. Esse tipo de metodologia é importante, pois é a partir dele que são enfatizadas interpretações em contexto e procuram representar pontos conflitantes entre outras situações (ANDRÉ, 2013).

Por fim, a Metodologia Científica é o estudo dos métodos ou dos instrumentos necessários para a elaboração de um trabalho científico. Ela aborda as principais regras para uma produção científica, fornecendo as técnicas, os instrumentos e os objetivos para um melhor desempenho e qualidade de um trabalho (MARCONI; LAKATOS, 2003). Apesar de inúmeras vantagens que essa metodologia traz para a área acadêmica, ela nos permite recolher dados no momento em que estão a acontecer, sem criar situações artificiais (SOARES, et. al. 2017). 0 grande número de pesquisas com esse tipo de metodologia se deu por se tratar de trabalhos com o uso de seres vivos relacionados a situações naturais, sem supor ou criar aspectos artificiais.

Dentre todas as amostras, foram identificados diferentes tipos de "documentos", o qual é definido como o tipo de publicação eletrônica e em que portal ela está localizada (Artigo Científico, Dissertação ou Tese). Partindo da separação e organização das amostras, foram encontrados 32 artigos publicados no Portal da CAPES e o restante da BDTD, sendo elas, 38 teses ou dissertações. Por isso, contabiliza-se todos os 32 artigos como artigos, propriamente ditos e o restante dividido entre teses e dissertações: 15 e 23 documentos, respectivamente. A seguir, tabela 3 representando esses resultados.

Tabela 3: Organização de acordo com o tipo de documento

\begin{tabular}{ccc}
\hline TIPO DE DOCUMENTO & No DE PUBLICAÇÕES & PERCENTUAL \% (aprox.) \\
\hline Artigo & 32 & 45,7 \\
Tese & 15 & 21,4 \\
Dissertação & 23 & 32,9 \\
\hline
\end{tabular}

Fonte: Obtidos pelo levantamento no Portal Periódico CAPES e BDTD, adaptado pelo autor, 2019.

Tanto a dissertação quanto a tese são trabalhos apresentados na conclusão de ciclos acadêmicos, estando o primeiro associado ao mestrado e o segundo ao doutorado. No entanto, a grande diferença entre as duas é o grau de complexidade e ineditismo, visto que o tempo de realização de ambas é diferente (FORTE; LEITE, 2008).

Uma tese, obrigatoriamente, deve aprofundar-se na pesquisa de um tema, saindo de superficialidades encontradas em dissertações que, por sua vez, não exigem alto grau de ineditismo. Isso acontece porque o tempo de formulação de uma tese é o dobro da de uma dissertação. Essa principal diferença foi encontrada nas amostras coletadas (FORTE; LEITE, 2008).

O artigo, por sua vez, é um tipo de documento que "não encerra ciclos" e pode ser produzido 


\section{ANAP

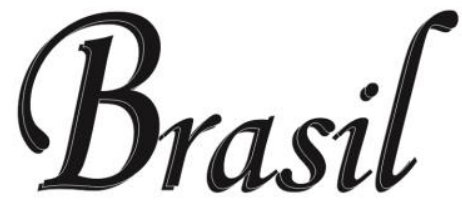 \\ ISSN 1984-3240 v. 12, n. 27}

durante uma tese ou dissertação. A pesquisa científica tem por objetivo estudar problemas relacionados a um determinado tema com o intuito de contribuir, assim como aprimorar para sociedade científica com a implantação e implementação de técnicas e métodos relativos as novas descobertas científicas (CASTIEL; VALERO; CYTED, 2007). No caso de estudos sobre Bioindicação, esse tipo de documento é extremamente importante, pois é a partir dele que providências de prevenções podem ser tomadas, evitando desastres irreversíveis, visto que a publicação do mesmo passa por rigorosas correções.

Exposto isso, vale ressaltar que nenhuma se posiciona melhor que a outra, mas sim que uma contribui mais que a outra no âmbito acadêmico (FORTE; LEITE, 2008).

Partindo para a parte técnica, foram encontrados documentos com vários tipos de bioindicador. São eles: animais, vegetais, microrganismos, líquens ou animais e vegetais, como disponíveis na Tabela 4. Então, foi observado que, em quase $50 \%$ das amostras, os bioindicadores de origem animal foram os mais utilizados. Seguinte dele encontram-se empatados os bioindicadores vegetais e os microrganismos, apresentando 12 artigos cada. $\mathrm{Na}$ mistura de animais e vegetais como indicadores biológicos foram encontrados apenas 4 textos, enquanto que os líquens foram utilizados em 8.

Tabela 4: Publicações de acordo com o tipo de bioindicador utilizado

\begin{tabular}{ccc}
\hline TIPO DE BIOINDICADOR & No DE PUBLICAÇÕES & PERCENTUAL \% (aprox.) \\
\hline Animal & 34 & 48,6 \\
Animal e Vegetal & 4 & 5,7 \\
Vegetal & 12 & 17 \\
Microrganismo & 12 & 17 \\
Líquens & 8 & 11,4 \\
\hline
\end{tabular}

Fonte: Obtidos pelo levantamento no Portal Periódico CAPES e BDTD, adaptado pelo autor, 2019.

Segundo Guimarães et. al. 2000, os resultados de experimentos com vegetais podem, sim, ser utilizados para comparar futuros efeitos em seres humanos, visto que esses bioindicadores possuem alta sensibilidade a elementos poluentes, mesmo que em pouca quantidade, sejam eles na água, no ar ou no solo. Assim, é possível dizer que quando elementos poluentes causam efeitos nulos ao organismo vegetal, o mesmo não afetará os demais seres vivos, incluindo os humanos.

Assim como acontece com todos os outros seres vivos, a bioindicação com o uso de animais é, também, muito eficiente, pois qualquer alteração pode comprometer toda uma comunidade ecológica e desequilibrá-la (CALISTO; GONÇALVES; MORENO, 2018). A maioria das pesquisas encontradas com o uso desses tipos de organismos biológicos apontou um levantamento de metodologias quantitativas, ou seja, conforme a ocorrência dos mesmos nos locais com determinada poluição. Por se tratar desse tipo de método onde não é necessário uma análise qualitativa, o número de publicações foi maior que o dos outros tipos.

Seguinte, a avaliação de áreas poluídas utilizando microrganismos também foi tida no presente artigo, sejam essas áreas: solo, água e ar. Os microrganismos constituem grande e dinâmica 


\section{ANAP

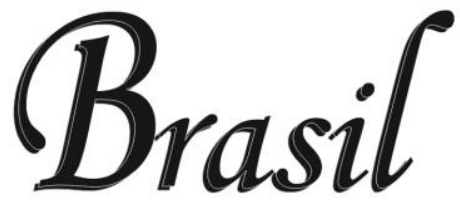 \\ ISSN 1984-3240 v. 12 , n. 27}

fonte e depósito de nutrientes em todos os ecossistemas e participam ativamente em processos benéficos no meio ambiente. Além disso, dentre as várias justificativas para o uso desses organismos como indicadores de qualidade ambiental, destacam-se a sua capacidade de responder rapidamente a mudanças no ambiente derivadas de alterações antrópicas (TÓTOLA; CHAER, 2002). Vale acrescentar que os microrganismos são vetores de disseminação de doenças em seres vivos, seja pela água, pelo ar, ou pelo solo (STERZ; GOMES; ROSSI, 2011), o que acarreta uma importância considerável de estudos.

Por último, os líquens. Esses organismos foram, particularmente encontrados em documentos que dizem respeito à qualidade do ar, pois são indicadores extremamente sensíveis ao ar e só conseguem se desenvolver se o mesmo estiver totalmente (ou em grande parte) puro, uma vez que são dependentes deste elemento para adquirirem nutrientes (CARNEIRO, 2004).

Todos os documentos que apresentaram o uso de dois bioindicadores (animais e vegetais) para um mesmo fim apresentaram tanto metodologia quantitativa, quanto qualitativa.

Por fim, definidos os bioindicadores utilizados em todas as amostras, a tabela 5 a seguir aponta as aplicações dos bioindicadores, ou seja, em qual local eles reagem à impactos ambientais. Foram encontrados 34 amostras que utilizam indicadores biológicos na água, 16 no ar, 12 no solo e 8 documentos que usufrui de um mesmo indicador para aplicar tanto na água, como no solo e no ar.

Tabela 5: Organização de acordo com a aplicação do bioindicador

\begin{tabular}{ccc}
\hline APLICAÇÃO DO BIOINDICADOR & No DE PUBLICAÇÕES & PERCENTUAL \% (aprox.) \\
\hline Água & 34 & 48,6 \\
Ar & 16 & 22,8 \\
Solo & 12 & 17 \\
Água, ar e solo & 8 & 11,4 \\
\hline
\end{tabular}

Fonte: Obtidos pelo levantamento no Portal Periódico CAPES e BDTD, adaptado pelo autor, 2019.

Nas últimas décadas, os ecossistemas aquáticos têm sido alterados de maneira significativa em função de múltiplos impactos ambientais advindos de atividades antrópicas, tais como mineração; construção de barragens e represas; retilinização e desvio do curso natural de rios; lançamento de efluentes domésticos e industriais não tratados; desmatamento e uso inadequado do solo em regiões ripárias e planícies de inundação; superexploração de recursos pesqueiros; introdução de espécies exóticas, entre outros. Como consequência destas atividades, tem-se observado uma expressiva queda da qualidade da água e perda de biodiversidade aquática, em função da desestruturação do ambiente físico, químico e alteração da dinâmica natural das comunidades biológicas (GOULART; CALLISTO, 2010). Relacionando o escrito acima com as amostras encontradas, nota-se que a água, por ser um recurso finito, as preocupações podem ser maiores e, por isso, há um número maior de pesquisas referentes à este elemento. Além disso, os bioindicadores utilizados foram tanto os macro como os microrganismos, resultando numa viabilidade maior de experimentos.

Seguinte dela, a poluição do solo está diretamente ligada a produção agrícola, visto que a 
problemática é conectada com a contaminação parcial ou completa do solo, comprometendo o fornecimento de nutrientes para a agricultura (ABREU Jr, et. al., 2005). Por esse motivo, os bioindicadores especificamente utilizados em avaliações de solo encontrados nos documentos amostrados são organismos capazes de fazer decomposição.

Porém, além da poluição da água e do solo, outro elemento se destaca quando nos referimos a poluição: o ar. Este, se torna tão importante quanto os outros visto que ele está presente no ambiente onde o ser humano capta oxigênio. $O$ ar contaminado, seja com gases tóxicos ou partículas microscópicas em suspensão, também não pode ser confinado em um determinado espaço, como o solo e a água. Assim, a contaminação do ar tem consequências diretas na vida do homem (NASS, 2013), justificando o número de amostras relacionadas à poluição atmosférica que foi relativamente alta.

Documentos que abordaram as três áreas potencialmente poluídas, contaram mais com indicadores microbiológicos e animais com vegetais, pois estes podem ser utilizados para todos as áreas e fins.

\section{Conclusão}

Diante do exposto, pode-se concluir que as pesquisas acadêmicas de todas as regiões e suas particularidades, devem sempre estar em constante produção, visto que são importantes para a prevenção e providências necessárias ao ambiente. Os elementos encontrados como "Ambientes poluídos" mostram que é necessário qualquer e todo tipo de cuidado com áreas suscetíveis a poluição, já que, se poluídas, podem causar danos irreversíveis à humanidade. Tal cuidado pode ser feito a partir de metodologias naturais, como os bioindicadores. Além disso, ressalta-se que todo tipo de estudo é válido e contribui com o desenvolvimento da Ciência na área acadêmica e, ainda que, que a ferramenta utilizada no presente trabalho possibilita o entendimento mais amplo sobre o tema abordado de forma a contribuir em produções científicas subsequentes.

\section{Referências Bibliográficas}

ABREU Jr, C. H. A.; BOARETTO, A. E.; MURAOKA, T.; KIEHL, J. C. Uso agrícola de resíduos orgânicos potencialmente poluentes: Propriedades químicas do solo e produção vegetal. 2005. Tópicos Ci. Solo, 4: 391-470. Piracicaba / SP. Disponivel em: <https://www.researchgate.net/publication/267968223>. Acesso em: 26 abr. 2019.

ANDRÉ, M. E. D. A. Estudo de Caso: Seu potencial na educação. 2013. Cad. Pesquisa. (49): 51-54. Rio de Janeiro. Disponível em: <http://publicacoes.fcc.org.br/ojs/index.php/cp/article/view/1427/1425>. Acesso em: 20 abr. 2019.

BHONA, F. M. C.; LOURENÇO, L. M.; BRUM, C. R. S. Violência doméstica: um estudo bibliométrico. 2011. Versão Online ISSN 1809-5267. Arq. bras. psicol. vol.63 no.1 Rio de Janeiro, RJ. Disponível em: $<$ http://pepsic.bvsalud.org/scielo.php?script=sci arttext\&pid=S180952672011000100010>. Acesso em: $20 \mathrm{mar}$. 2019.

BRASIL. Ministério do Meio Ambiente. Disponível em: <www.mma.gov.br>. Acesso em: 20 abr. 2019.

CAIRNS Jr., J.; MCCORMICK, P. V.; NIEDERLEHNER, B. R. A proposal framework for developing indicators of 


\section{ANAP

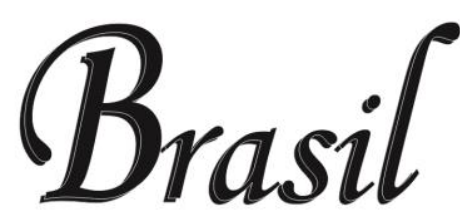 \\ ISSN 1984-3240 v. 12, n. 27}

ecosystem health. Hydrobiologia, 263 p.1-44. Netherlands: Publishen:Springen, 1993. Available from: < https://link.springer.com/article/10.1007/BF00006084>. Cited in: 10 mar. 2019.

CALISTO, M.; GONÇALVES, J. F.; MORENO, P. Invertebrados Aquáticos como Bioindicadores. 2018. Univerdade Federal de Minas Gerais. Disponível em: <https://manuelzao.ufmg.br/wpcontent/uploads/2018/08/invertaquaticos.pdf>. Acesso em: 24 abr. 2019.

CARDOSO, K. M.; PAULA, A.; SANTOS, J. S.; SANTOS, M. L. P. Uso de espécies da arborização urbana no Biomonitoramento de poluição ambiental. 2017. Rev. Ciência Florestal. vol. 27 no.2 Santa Maria, RS. Disponível em: $\quad<$ http://www.scielo.br/scielo.php?script=sci arttext\&pid=S198050982017000200535\&lng=en\&t|ng=en>. Acesso em: 11 abr. 2019.

CARNEIRO, R. M. A. Bioindicadores vegetais de poluição atmosférica: uma contribuição para a saúde da comunidade. Dissertação de Mestrado apresentada à Escola de Enfermagem de Ribeirão Preto / USP. 2004. Ribeirão Preto - SP. Disponível em: <http://www.teses.usp.br/teses/disponiveis/22/22133/tde-19102004170613/pt-br.php>. Acesso em: 06 abr. 2019.

CASTIEL, L.D.; VALERO, J. S.; CYTED, R. M. Entre fetichismo e sobrevivência: $\mathrm{O}$ artigo científico é uma mercadoria acadêmica?. 2007. Caderno Saúde Pública, Rio de Janeiro, RJ. 23(12):3041-3050. Disponível em: <https://www.scielosp.org/pdf/csp/2007.v23n12/3041-3050/pt>. Acesso em: 20 abr. 2019.

CAVALCANTI, C.; BEGOSSI, A. Meio ambiente, desenvolvimento sustentável e políticas públicas. In: Curso Agenda 21, 1997. Disponível em: <institutoembratel.org.br>. Acesso em: 11 abr. 2019.

FACURY, D. M.; CARVALHO, V. J. B. B.; COTA, G. E. M.; MAGALHÃES Jr, A. P. Panorama das publicações científicas sobre o rompimento da Barragem de Fundão (Mariana-MG): subsídios às investigações sobre o maior desastre ambiental do país. 2019. Caderno de Geografia, v.29, n.57. Belo Horizonte, MG. Disponível em: <http://periodicos.pucminas.br/index.php/geografia/article/view/19970/14479>. Acesso em: 08 mai. 2019.

FIALHO, R. C. O uso de Bioindicadores vegetais no monitoramento e controle da poluição atmosférica: A importância dos Bioindicadores. Informativo técnico publicado pela Associação dos Engenheiros da CETESB - ASEC e pelo Conselho de Representantes dos Funcionários da CETESB - CRF, para distribuição interna e limitada aos empregados da SMA/CETESB. v. $2 . \quad$ n. 4.42002 .4 Disponível em: <http://www.teses.usp.br/teses/disponiveis/22/22133/tde-19102004170613/publico/mestrado.pdf>. Acesso em: 09 mar. 2019.

FORTE, S. H. A. C.; LEITE, F. T. Manual de elaboração de Tese, Dissertação e Monografia. 2008. Fundação Edson Queiroz. Universidade de Fortaleza. Fortaleza, CE. Disponível em: <http://hp.unifor.br/pdfs notitia/2269.pdf >. Acesso em: 20 abr. 2019.

GUIMARÃES, E. T.; DOMINGOS, M.; ALVES, E. S.; CALDINI JR, N.; LOBO, D. J. A.; LICHTENFELS, A. J. F. C.; SALDIVA, P. $\mathrm{H}$. N. Detection of the genotoxicity o fair pollutants in and around the city of São Paulo (Brazil) with Tradescantiamicronucleus (TRAD-MCN) assay. 2000. Environmental and Experimental Botany. V.44, n. 1, p. 1-8. Available in: <https://www.ncbi.nlm.nih.gov/pubmed/10927123>. Cited in: 20 abr. 2019.

KAPUSTA, S. C. Bioindicação ambiental. Biblioteca da UFSC. Escola Técnica da Universidade Federal do Rio Grande do Sul. 2008. Porto Alegre - $\quad$ RS. Disponível em: $<$ http://proedu.rnp.br/bitstream/handle/123456789/716/Bioindicacao PB.pdf?sequence=3\&isAllowed $=\mathrm{y}>$. Acesso em: 09 mar. 2019.

KLUMPP, A.; ANSEL, WOLFGANG.; KLUMPP, G.; FOMIN, A. Um novo conceito de monitoramento e comunicação ambiental: A rede Européia para a avaliação da qualidade do ar usando plantas Bioindicadoras (EuroBionet). Revista Brasil. Bot. V.24, n.4 (suplemento), p.511-518, 2001. São Paulo - SP. Disponível em: <http://www.scielo.br/pdf/\%0D/rbb/v24n4s0/9472.pdf>. Acesso em: 06 abr. 2019. 


\section{ANAP

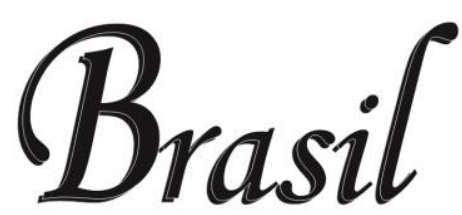

LIMA, J. S. Processos biológicos e o biomonitoramento: Aspectos bioquímicos e morfológicos. In: MAIA, N.B.; MARTOS, H. L.; BARRELLA, W. (Org.). Indicadores ambientais: Conceitos e aplicações. São Paulo: Editora da PUC. p. 77-94. 2001. Disponível em: <http://www.techoje.com.br/site/techoje/categoria/abrirPDF/172>. Acesso em: 11 mar. 2019.

LOMBARDO, M. A. Ilha de Calor nas Metrópoles: O exemplo de São Paulo. 1985. São Paulo: Hucitec.

MARCONI, M. A.; LAKATOS, E. M. Fundamentos da Metodologia Científica. 2013. Editora Atlas SA. 5a Ed. Disponível em: $<$ https://docente.ifrn.edu.br/olivianeta/disciplinas/copy of historia-i/historia-ii/china-e-india>. Acesso em: 20 abr. 2019.

NASS, D. P. O conceito de poluição. 2013. Instituto de Química de São Carlos da Universidade de São Paulo. São Carlos / SP. Disponível em: mirtes.webnode.com/200000113738c57486a/O\%20conceito\%20de\%20polui\%C3\%A7\%C3\%A3o.pdf>. Acesso em: 26 abr. 2019.

NIMIS, P. L.; LAZZARIN, G.; LAZZARIN, N.; SKERT, N. Biomonitoring of trace elements with lichens in Veneto (NE Italy). The Science of the Total Environment, $v 255$, p.97 - 111. 2000. Available in: <https://www.sciencedirect.com/science/article/pii/S004896970000454X>. Cited in: 02 abr. 2019.

PORTILHO, F. Ambientalização do Consumo: Alianças entre Movimentos Ambientalistas e Movimentos de Defesa dos Consumidores. In: III Encontro da ANPPAS, 2006, Brasília-DF. Disponível em: <anppas.org.br>. Acesso em: 06 mar. 2019.

SATARUG, S.; GARRET, S. H.; SENS, M. A.; SENS, D. A. Cadmium, environmental exposure, and health outcomes. 2010. Environmental Health Perspectives, North Columbia, v. 118, no. 2. Disponível em: <https://www.ncbi.nlm.nih.gov/pubmed/20123617>. Acesso em: 11 abr. 2019.

SIDONI, O. J. G.; HADDAD, E. A.; MENA-CHALCO, J. P. A ciência nas regiões brasileiras: evolução da produção e das redes de colaboração científica. 2016. Rev. TransInformação. 28(1):15-31. Campinas. Disponível em: <http://www.scielo.br/pdf/tinf/v28n1/0103-3786-tinf-28-01-00015.pdf>. Acesso em: 11 abr. 2019.

SOARES, A.; CAROLINE, A.; CARLOS, L.; BARROS, P.; MARTINS, T. Método de Observação. 2017. UNIR, Rondônia. Disponível em: <http://www.pi.unir.br/uploads/36315994/arquivos/2 1609242645.pdf>. Acesso em: 20 abr. 2019.

STERZ, C.; GOMES, M. F. R.; ROSSI, E. M. Análise microbiológica e avaliação de macroinvertebrados bentônicos como bioindicadores da qualidade da água do Riacho Capivara, município de Mondaí, SC. 2011. Unoesc \& Ciência - ACBS, Joaçaba, v. 2, n. 1, p. 1 7-16. Disponível em: <https://portalperiodicos.unoesc.edu.br/acbs/article/view/735/pdf 132>. Acesso em: 27 abr. 2019.

TORGAL, F. P.; JALALI, S. Construção Sustentável: O caso dos materiais de construção. In: Congresso Nacional, 3. 2007. Coimbra-PT. Disponível em: <http://repositorium.sdum.uminho.pt/bitstream/1822/7542/1/Artigo\%204.pdf>. Acesso em: 20 abr. 2019.

TÓTOLA, M. R.; CHAER, G. M. Microrganismos e processos microbiológicos como indicadores da qualidade dos solos. In: ALVAREZ V., V. H. et al. (Ed.). Tópicos em ciência do solo. Viçosa, MG: Sociedade Brasileira de Ciência do Solo, 2002. v. 2. p. 195-276.

WEBSTER, J.; WATSON, J. T. Analyzing the past to prepare for the future: writing a literature review. 2002. MIS Quarterly \& The Society for Information Management, v.26, n.2, pp.13-23. Available in: <https://www.jstor.org/stable/4132319?seq=1\#page scan tab contents >. Cited in: 20 abr. 2019. 